\title{
Applications of high temperature superconductors
}

T.M. Silver, S.X. Dou and J.X. Jin

Institute for Superconducting and Electronic Materials,

University of Wollongong, Wollongong, NSW 2522, Australia

Tost of us are familiar with the basic idea of superconductivi$M_{\text {ty, that a superconductor can carry a current indefinitely in a }}$ closed loop, without resistance and with no voltage appearing. In a normal metal, such as copper, the free electrons act independently. They will move under the influence of a voltage to form a current, but are scattered off defects and impurities in the metal. This scattering results in energy losses and constitutes resistance. In a superconducting metal, such as niobium, resistance does not occur, because under the right conditions the electrons no longer act as individuals, but merge into a collective entity that is too large to 'see' any imperfections. This collective entity, often described as a Bose condensate, can be described by a single macroscopic quantum mechanical wave function. Similar condensates are behind the peculiar behaviour seen in superfluid helium and in very cold gases of trapped rubidium atoms.

\section{History of superconductivity}

Superconductivity is not a new phenomenon. H. KammerlinghOnnes first demonstrated it in 1911 with his finding that resistance dropped to zero in a sample of mercury held at or below $4 \mathrm{~K}$. This sudden drop in resistance, shown in Fig. 1, is characteristic of all superconductors. A number of metals besides mercury (including tin and lead, but not including magnetic metals or very good normal conductors such as copper and gold), as well as certain alloys and intermetallic compounds were also soon found to be superconducting at similar low temperatures. Above the transition or critical temperature $T_{c}$ the metal or alloy behaved as a normal conductor. Even below the critical temperature, the material would revert to normal if a critical magnetic field or critical current were exceeded. The surface plotted in Fig. 2 defines the critical temperature, field and current for a bismuth-based superconductor. Until 1986 the record critical temperature was $23 \mathrm{~K}$ for $\mathrm{Nb}_{3} \mathrm{Ge}$. Two classes of superconductor were recognised. Type I, mostly the pure metals, had an abrupt transition from the superconducting to the normal state. Type II superconductors also allowed for a mixed state with normal and superconducting regions coexisting. Other important superconductor properties were discovered during the course of the last century, such as the exclusion of magnetic flux lines (the Meissner effect), which is responsible for magnetic levitation, as shown in Fig. 3, and quantum mechanical tunnelling over a narrow gap between two superconductors (the Josephson effect), which is used in extremely sensitive magnetometers.

The discovery of superconductivity soon generated interest in practical applications, mainly because of its potential to save energy. Indeed the replacement of copper or other normal conductors by superconducting materials avoids heat dissipation and other energy losses due to finite resisitance. In some types of equipment such as magnetic separators, these losses may account for most of the energy consumed in the device. Early prototypes for motors, transmission lines and energy storage magnets were developed, but they were never widely accepted. There were important reasons for this, apart from the tremendous investment in existing technology. In most superconducting metals and alloys the superconductivity tends to fail in self-generated magnetic fields when the current densities through them are increased to practical levels. A second problem was the cost and complexity of operating refrigeration equipment near liquid helium temperatures $\left(4 \mathrm{~K},-269^{\circ} \mathrm{C}\right)$. Removing one watt of heat generated at $4 \mathrm{~K}$ demands about $1000 \mathrm{~W}$ of refrigeration power at room temperature

While applications were stalled, progress was still being made in theoretical models. The London theory (1935) and the subsequent Ginzburg-Landau (GL) theory including quantum effects were phenomenological. These theories did not attempt to explain superconductivity on a microscopic level, but achieved good success in describing supercurrent behaviour. The BCS theory (1957) of Bardeen, Cooper and Schrieffer was a detailed microscopic theory that was quickly accepted as an explanation for the condensate in the superconductors that were known at the time. Such a condensate requires that the particles composing it be bosons, that is, have integral spin. Bosons obey Bose-Einstein statistics. Below the critical temperature the bosons in a superconductor can all gather together in the lowest possible energy state to form the condensate, and the greater the number that have accumulated, the harder it is for one of them to leave. Electrons are not bosons because they do not have integral spin. BCS theory

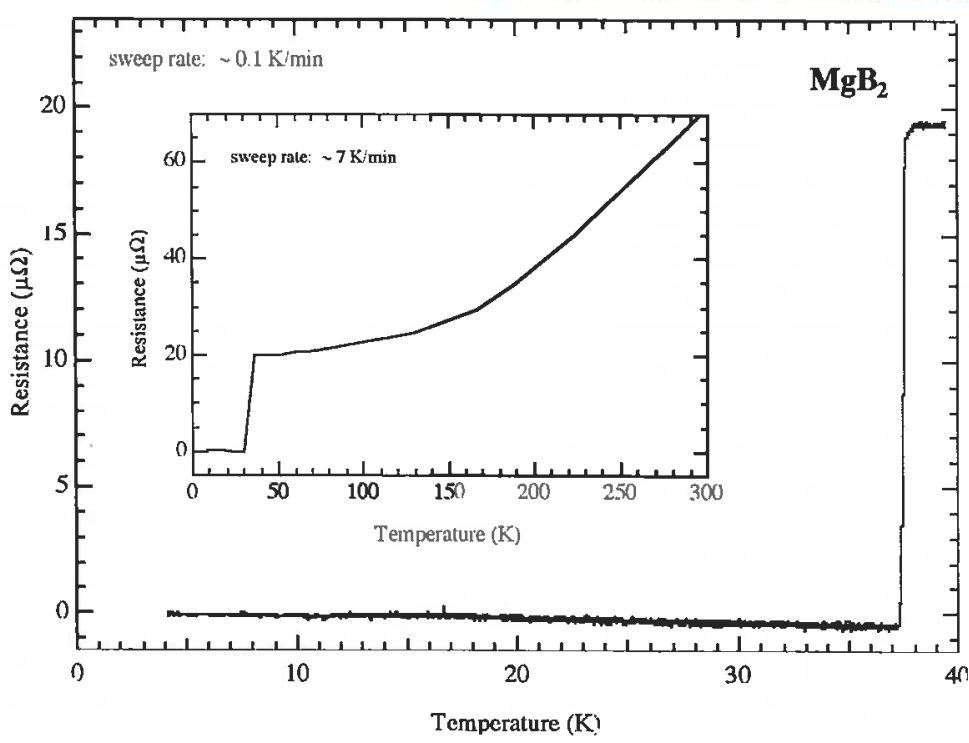

Fig. 1: A drop to zero resistance at the critical temperature is seen in all superconductors, including the newly discovered superconductor $\mathrm{MgB}_{2}$. Figure courtesy of R.A. Lewis and A.D. Martin, ISEM. 


\section{Superconductivity of Bi-2212 T'ape Sample}

Je $\left(\mathrm{A} / \mathrm{cm}^{2}\right)$

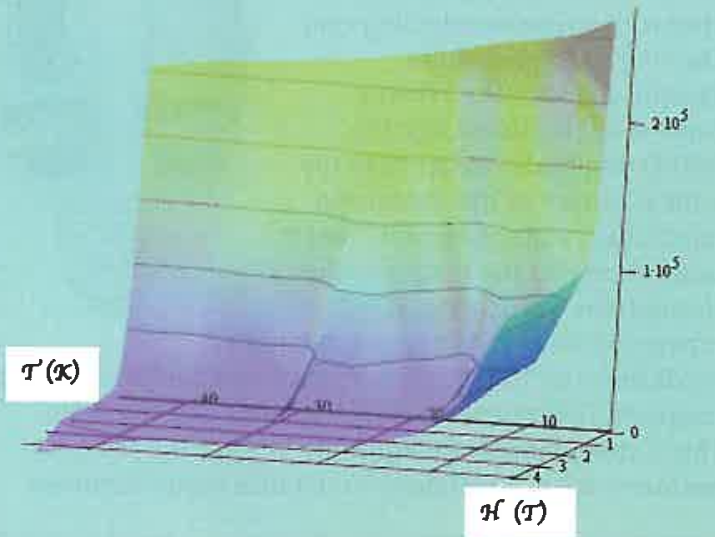

Fig. 2: The critical surface shown here defines the critical current $(\mathrm{J})$ of a bismuth based superconducting tape for various values of magnetic field $(H)$ and temperature $(T)$. If the current is raised above the surface value at a particular field and temperature, superconductivity is lost, and the tape behaves as a normal conductor. Data courtesy of Mikhail lonescu, ISEM.

explained how the interaction between the electrons and the phonons or lattice vibrations in the metal causes an electron-electron attraction. Some of the electrons form so-called Cooper pairs where the spins and momentum are opposite and therefore cancel out. Because the Cooper pairs have zero spin, they can participate in Bose condensation. It appeared that superconductivity was well explained and only possible at very low temperatures.

\section{The first practical superconductors}

In 1961 Kunzler et al. opened the "Type II" era by drawing a Nb tube filled with $\mathrm{Nb}_{3} \mathrm{Sn}$ powder into a wire with a current density $\mathrm{J}_{\mathrm{c}}$ of $\sim 10^{5} \mathrm{~A} / \mathrm{cm}^{2}$. The wire remained superconducting in fields up to $8.8 \mathrm{~T}$. It was soon discovered that other highly cold worked type II alloys such as $\mathrm{Nb}$ - Ti and $\mathrm{Nb}-\mathrm{Zr}$ were also able to carry high transport currents at high, technologically useful magnetic fields. In a type II superconductor there are two critical temperatures (or magnetic fields at a given temperature). The lower one indicates the onset of the mixed state as magnetic flux lines penetrate the material, with each accompanied by a vortex of supercurrent surrounding a normal state core. The higher one indicates the complete loss of superconductivity as the vortex cores overlap. In the cold-worked "hard" or "dirty" superconductors there are a wide range of defects and impurities that act as pinning centres to trap the flux lines and vortices and prevent further penetration and loss of superconductivity. The "dirty" superconductors had considerable potential for technological applications despite the continuing problems of working near liquid helium temperature. This was particularly true for magnet applications, because much stronger magnetic fields could be achieved as compared to conventional electromagnets. It was even possible to magnetise a superconducting magnet from a current source, then detach and short-circuit the winding, leaving what was effectively a giant permanent magnet that would hold its magnetic field constant for months.

There are a number of extra high field magnets, transmission cables and generators that have been designed and built using

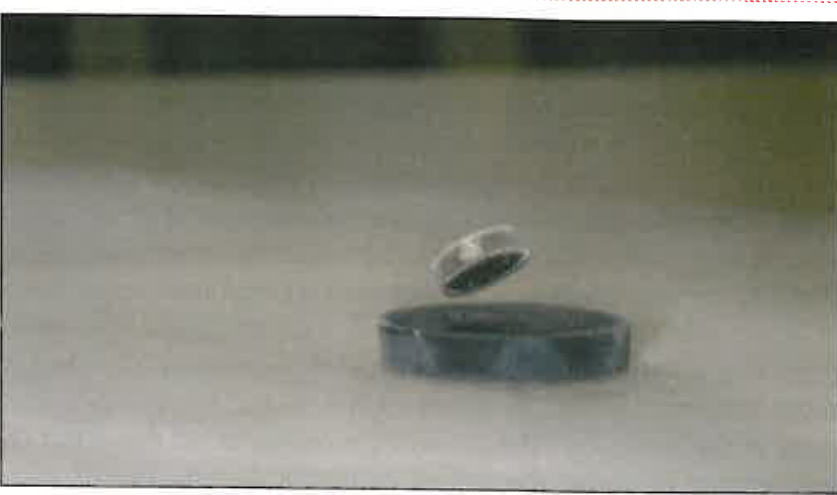

Fig. 3: Superconductors exclude magnetic flux (the Meissner effect). A magnet lowered towards a superconductor will 'see' an identical magnet the same distance below the surface due to supercurrents induced on the superconductor surface. The resultant repulsive force levitates the magnet, provided that the force is greater than the magnet's weight. Magnetic levitation (Maglev) trains and frictionless superconductor bearings use this repulsive force. Here a neodymium-iron-boron magnet floats above a disk of lead-doped BSCCO superconductor, with a critical temperature $T_{c}$ of $108 \mathrm{~K}$. The superconductor has been cooled in liquid nitrogen, and the magnet falls when the superconductor is warmed above $T_{c}$.

these low critical temperature (LTS) superconductors. Such superconductors are commonly used in the construction of the powerful magnets and electric resonators found in big particle accelerators such as CERN and Fermilab. The electric resonators produce very strong oscillating fields for accelerating the particles, while the magnets focus them and bend their path. The present commercial LTS superconductors for large-scale applications are typically $\mathrm{NbTi}$ or $\mathrm{Nb}_{3} \mathrm{Sn}$ superconducting strands embedded in a copper matrix.

\section{The high temperature superconductors}

The high temperature superconductors (HTS) appeared on the scene in 1986 when Bednorz and Miller discovered a new oxide ceramic superconductor $(\mathrm{La}, \mathrm{Ba})_{2} \mathrm{CuO}_{4}$, with a critical temperature of $35 \mathrm{~K}$. High temperature is relative. New HTS oxides were quickly discovered. The record HTS critical temperature is now $\sim 133.5 \mathrm{~K}$ (160 K at high pressure) for $\mathrm{HgBa}_{2} \mathrm{Ca}_{2} \mathrm{Cu}_{3} \mathrm{O}_{8+\mathrm{x}}$. Most current work is focussed on two families of materials, $\mathrm{YBCO}$ $\left(\mathrm{YBa}_{2} \mathrm{Cu}_{3} \mathrm{O}_{7-\mathrm{x}}, \mathrm{T}_{\mathrm{c}} \sim 92 \mathrm{~K}\right)$ and BSCCO $\left(\mathrm{Bi}_{2} \mathrm{Sr}_{2} \mathrm{Ca}_{1} \mathrm{Cu}_{2} \mathrm{O}_{8+\mathrm{x}}(\mathrm{Bi}-\right.$ 2212), $\mathrm{T}_{\mathrm{c}} \sim 85 \mathrm{~K}$, and $\mathrm{Bi}_{2} \mathrm{Sr}_{2} \mathrm{Ca}_{2} \mathrm{Cu}_{3} \mathrm{O}_{10+\mathrm{x}}(\mathrm{Bi}-2223), \mathrm{T}_{\mathrm{c}} \sim 110 \mathrm{~K}$, shown in Fig. 3). The crystal structures of these materials can all be described as oxygen-deficient multiple perovskites with $\mathrm{CuO}_{2}$ planes. There is always a strong anisotropy or direction sensitivity in the superconducting behaviour. Effectively the supercurrents flow along the $\mathrm{CuO}_{2}$ planes, which are linked together by Josephson coupling. The HTS materials can be classed as type II superconductors. Their behaviour is well described by the London and GL theories, but there is still no generally accepted microscopic theory for high temperature superconductivity. The superconducting properties of $\mathrm{MgB}_{2}$, a new high temperature superconductor that is not a copper oxide were just discovered this year by a team led by Jun Akimitsu at Aoyama-Gakuin University, Tokyo. The critical temperature is $39 \mathrm{~K}$, but research on this compound has just begun, and it is not clear whether it is simply an LTS type II superconductor with an unusually high critical temperature or represents a whole new superconductor family. 
The discovery of the HTS materials aroused tremendous excitement, because many of them are superconducting and carry significant current above the boiling point of liquid nitrogen at $77.4 \mathrm{~K}\left(-196^{\circ} \mathrm{C}\right)$. It appeared as though a whole new age was opening up for practical applications, particularly large-scale applications. Because the mechanisms for the new type of superconductivity were not well understood, it seemed that room temperature superconductivity was just around the corner. Even at liquid nitrogen temperatures enormous savings are possible. Removing a watt of heat generated at $77 \mathrm{~K}$ requires $10 \mathrm{~W}$ or less at room temperature (as opposed to $1000 \mathrm{~W}$ at $4 \mathrm{~K}$ ). The development of the cryocooler has allowed for refrigeration down to LTS temperatures without the use of cryogenic liquids, but if cryogenic liquids are used for cooling, a litre of liquid helium costs approximately $\$ 25$, as opposed to $60 \$$ for a litre of liquid nitrogen. It was expected that aluminium and copper would be completely replaced by HTS materials in electromagnets, generators, power transmission lines, motors, and magnetic energy storage. Maglev trains would float above their tracks and flywheels above their superconducting bearings, while computing would depend on ultra-fast Josephson junctions.

These grandiose expectations inevitably led to disappointment. Room temperature superconductivity has remained a dream. Critical current densities in HTS materials also tend to be naturally too low for technological applications, while there are persistent problems with poor mechanical properties. These problems are both related to the ceramic, granular, anisotropic nature of the HTS materials (other than $\mathrm{MgB}_{2}$, which behaves as a brittle metal). They need to be formed at high temperatures in the presence of oxygen. Like all ceramics, HTS materials are very brittle and very difficult to shape and handle, while long, flexible, superconducting wires are necessary for many large-scale applications. Large supercurrents can only flow along the $\mathrm{CuO}_{2}$ planes, and only a small fraction of the material in a completed device is likely to be correctly oriented. The grain boundaries attract impurities, leading to weak links, which reduce the inter-grain current density and provide an easy path for flux vortices to enter the material. Flux creep or vortex penetration into HTS material is unusually rapid. The coherence length or diameter of a vortex core tends to be very small. This is a problem because pinning is most effective if the defect or impurity is of the same 'size' as the coherence length.

\section{Small-scale applications of HTS superconductors}

Vast research efforts over the past 15 years have been aimed at overcoming these deficiencies. The most success has been achieved with HTS small-scale devices that rely on the special properties of superconductors, especially the Josephson quantum tunnelling effects. The materials for these devices have been largely perfected, although there are still problems in incorporating superconductors into integrated circuits. Progress is now largely limited by systems and refrigeration packages, not by materials, films or junctions. Some LTS and HTS small-scale devices, most notably SQUIDS, are now commercially available, with HTS devices, such as the one shown in Fig. 4, having the advantage of smaller size (due to the smaller refrigeration system required) and much smaller power consumption. YBCO is widely used for the HTS small-scale devices because it has a high critical temperature and can accommodate high current densities.

Perhaps the best-known small-scale device is the SQUID (Superconducting QUantum Interference
Fig. 4: A SQUID device, such as this HTS SQUID magnetometer from Tristan Technologies, contains a chip with a superconducting ring. The ring has one or more Josephson junctions. When a current is introduced into the SQUID ring that is larger than the critical current of the Josephson junctions, a voltage appears that is proportional to the magnetic flux through the SQUID ring. SQUID magnetometers can measure magnetic fields down to $10^{-14} \mathrm{~T}$ and are sensitive enough to pick up magnetic fields generated by the heart and the brain. This is three orders of magnitude better than the best performance of a standard inductance magnetometer.

Device) magnetometer. A SQUID contains a ring of superconductor with one or more Josephson junctions. When a current is introduced into the SQUID that is larger than the critical current of the Josephson junctions a voltage appears that is proportional to the magnetic flux through the SQUID ring. The SQUID is so exceedingly sensitive that it can detect magnetic fields 100 billion times smaller than the Earth's field, and it can also detect any other physical quantity that can be converted to a magnetic flux.

Other devices that have reached commercial availability are HTS passive RF and microwave filters for wide-band communications and radar. These are based on conventional microstrip and cavity designs with superconductors used for the microstrips or to line the metal cavity. Both types are shown in Figure 5. They have the advantages of very low noise and much higher selectivity and efficiency than conventional filters. This is offset by the disadvantage of the requirement for refrigeration, but for some applications they are still worthwhile. RSFQ (Rapid Single Flux Quantum) logic for integrated circuits and computers is still under development. The logic elements are composed of superconductor rings with a Josephson junction shunted by a resistor. The magnetic flux that can be contained in such a ring is quantised (i.e. comes in discrete packets). The logic functions are built around the very short voltage pulses that occur every time a flux quantum enters or leaves the ring. The pulses can be transmitted ballistically between gates over superconducting lines. Signals propagate virtually without dispersion. Because these elements dissipate hardly any power individual devices can be packed as close as $1 \mu \mathrm{m}$. RSFQ logic circuits are also have speeds of up to 100 times those of fast semiconductor ICs combined with great accuracy. Data rates of more than $750 \mathrm{~Gb} / \mathrm{s}$ have already been experimentally demonstrated. The first RSFQ device to be mar-

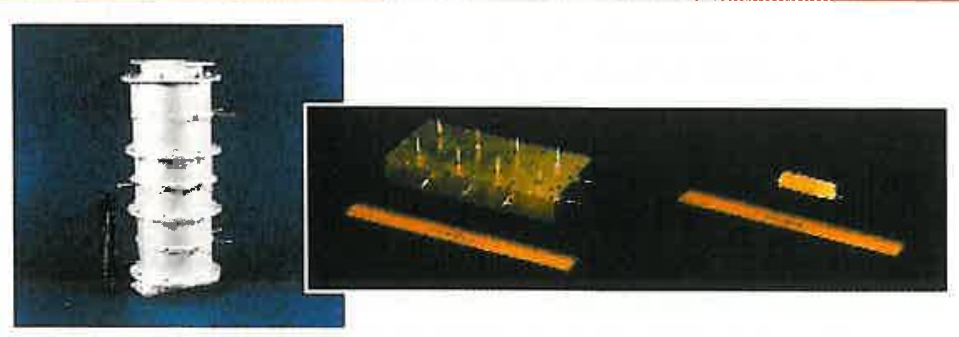

Fig. 5: Commercial HTS microwave filters from Conductus. The filter on the left is of a cavity type, while the others are microstrip filters. 
keted is likely to be an A/D converter. Niobium LTS superconductors are most widely used for RSFQ logic, but research is continuing on the problems of constructing integrated circuits using HTS materials. These largely arise from grain boundary effects and the tendency for underlying layers to be degraded in growing multi-layer circuits.

\section{Large-scale applications}

LTS superconductors are already in quite common use for large-scale applications, but still have stability problems and problems due to the complex and expensive refrigeration required. Large-scale applications for HTS materials present a major challenge to the materials scientist. Compared with the small-scale applications, a large-scale application generally means that much larger currents and lengths of superconductor are required in a working environment where the magnetic field may be several Teslas. The most important applications under consideration are magnets, power transmission cables, current leads, fault current limiters, transformers, generators, motors, and energy storage. In most of these applications, apart from fault current limiters, HTS superconductors would simply replace conventional conductors, e.g. a copper winding in an electromagnet is replaced by a BSCCO tape winding. Fault current limiters for electrical utilities depend on the fact that superconductivity is lost and resistance appears above a critical current. Under normal conditions the fault current limiter is superconducting and offers no impedance to the ordinary current. During a power surge the large fault current exceeds the critical current and is limited by the consequent resistance as the superconductor goes normal. The superconductivity returns after the current spike.

Applications related to magnet technology are probably among the most significant that are under research at the present time. These include magnetic energy storage, Maglev trains (relying on repulsion between magnets mounted on the train and the guideway) and magnets for MRI and other medical imaging applications. In all these cases the superconductor must not only carry a large current with zero resistance under a high magnetic field, but it must be possible to fabricate it in long lengths with high flexibility and a high packing density. This is because for large electromagnets it is necessary to build high current ampereturn windings. For the large-scale applications the critical current density $\mathrm{J}_{c}$ must generally be greater than $10^{4} \mathrm{~A} / \mathrm{cm}^{2}$, and the magnet must operate well in a magnetic field from about $0.2 \mathrm{~T}$ for a transmission cable to about $4 \mathrm{~T}$ for a generator. Figure 6 shows how some HTS superconductors meet the requirements of the different applications. The performance of the common LTS superconductor NbTi is also shown for comparison.

Research on HTS large-scale applications has focused on the BSCCO family because it is difficult to grow YBCO in bulk. Research has focused on processing methods, especially heat and pressure processing, to improve the phase and grain composition of precursor powders and to achieve greater density of the final product, thus reducing weak links. Selected defects and impurities are introduced to improve the flux pinning. While some applications such as current leads rely on bulk material, most

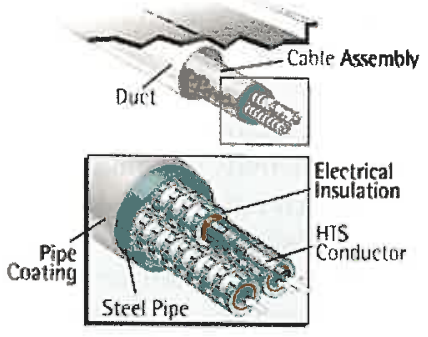

Figure 7(a)

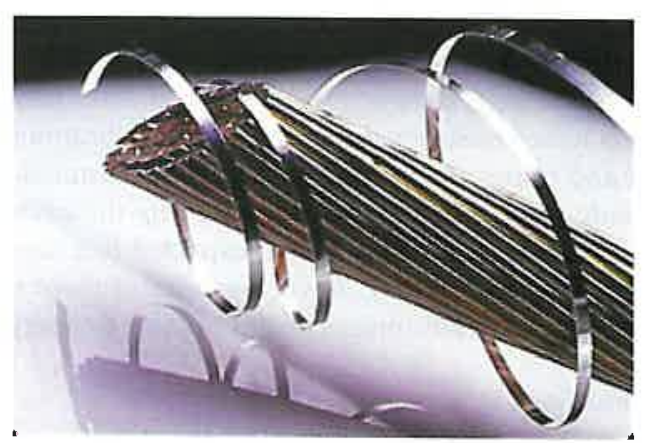

Figure 7(b)

Fig. 7: American Superconductor is supplying the superconducting power cables for the Detroit Edison project. The current is carried by silver sheathed BSCCO tape. The construction of one type of cable is shown in (a). Liquid nitrogen flows through the core of the cable to provide the necessary cooling. (b) compares the BSCCO tape with copper cable. Three such tapes can carry as much current as the copper cable. 
applications require very long, flexible tapes or wires. The powder in tube (PIT) method can achieve this. BSCCO powder is packed into a silver tube, which is drawn fine and goes through a sintering, rolling and annealing process. The individual BSCCO cored wires resulting from this process can be bundled together to create a multifilamentary tape. A number of large-scale prototype devices using BSCCO wire or tape have been constructed and shown to work satisfactorily. The tapes are cooled below $40 \mathrm{~K}$ when operation in high magnetic fields is required and to between 50 and $77 \mathrm{~K}$ for low field operation. The major remaining barrier to wider use is cost.

HTS underground power transmission cables carrying 3 to 5 times the current of a copper cable of the same diameter, as shown in Figure 7, are already coming into commercial use in cities such as Detroit. The extra cost of the HTS cable is justified because capacity can be upgraded using existing conduits and avoiding the cost and inconvenience of digging up the streets. Large (1000 hp) asynchronous ac motors with HTS armature windings are also very close to commercial viability. Their low losses and high magnetic fields allow for a much smaller and lighter motor in proportion to its output. Even after allowing for the energy required for refrigerating the HTS winding, such motors are approximately twice as efficient as the best conventional motors. BSCCO HTS current leads for LTS magnetic energy storage systems are also very close to viability. These have a current carrying capacity enormously greater than that of the copper leads they replace, so they not only eliminate losses due to resistance, but their small cross-section reduces heat transport into the LTS magnet. This allows for large energy savings on cooling.

\section{Conclusion}

The discovery of ceramic-type HTS materials in 1986, which exhibit superconductivity at liquid nitrogen temperature, has led to extensive research into materials formulation, characterisation and methods of fabrication. Many of the problems of fabrication into long lengths or thin films have been overcome, and more than 50 companies around the world have set out to commercialise HTS over the past 10 years. Small-scale devices have been commercially available for some time. The major problems for large-scale applications have been achieving high critical transport currents under high magnetic fields. The technical performance of long, state-of-the-art BSCCO wires have allowed prototypes to be constructed for large-scale applications, such as magnets and transmission cables, and HTS transmission cables have already come into commercial use where the advantages of high current density are crucial. It is expected that there will be further progress in bringing down the costs of these materials, leading to significant advantages in efficiency and energy saving.

\section{Further Reading}

D. Driscoll, V. Dombrovski, and B. Zhang 'Development status of superconducting motors', IEEE Power Engineering Review, May 2000, pp. 12-15.

W.V. Hassenzahl Applications of superconductivity to electric power systems', IEEE Power Engineering Review, May 2000, pp. 4-7.

H.S. Nalwa, ed. Handbook of Advanced Electronic and Photonic Materials and Devices. Volume 3: High Tc Superconductors and Organic Conductors (San Diego, Academic Press, 2001), pp. 129-168.

J.M. Rowell, 'Recommended directions of research and development in superconducting electronics', IEEE Transactions on Applied Superconductivity 9(2), 1999, pp. $2837-2848$. 